\title{
Implicit, Context Management Systems for Mobile Health Services
}

\author{
Vahid Farahmandian', Abbas Asosheh ${ }^{2}$ \\ ${ }^{1}$ Faculty of Medicine, Tarbiat Modares University, Tehran, Iran \\ ${ }^{2}$ Faculty of Electrical and Computer, Tarbiat Modares University, Tehran, Iran \\ Email: v.farahmandian@modares.ac.ir, asosheh@modares.ac.ir
}

Received 18 February 2015; accepted 8 March 2015; published 17 March 2015

Copyright (C) 2015 by authors and Scientific Research Publishing Inc.

This work is licensed under the Creative Commons Attribution International License (CC BY). http://creativecommons.org/licenses/by/4.0/

cc) (i) Open Access

\begin{abstract}
Objectives: In this paper data flow and executive model of Mobile Health services risk management by the use of context aware systems are provided. Materials and Methods: Mobile health (M-Health) refers to using portable electronic devices having application for delivering health services and patient's information management. M-Health can offer various services remotely in prevention, detection, control, and treatment of disease or in the conditions of disaster for a patient or an environment. These services can have more acceptable quality by the help of Context Aware Systems which are defined as the capacity of computing equipment for detection, feeling, interpreting, and replying to user's local environmental aspects and computing equipment itself. In this paper, executive model is offered for managing services of $\mathrm{M}$-Health based on context aware systems. One of the supplies of developing a context aware system is having a clear and well-defined definition of context and developing appropriate context information provider. In order to deliver high quality and well-managed M-Health services in the form of context aware systems, having clinical risk management plan is necessary. Conclusions: M-Health services need to develop appropriate communication strategies for interacting with stockholders at each stage of clinical risk management process. Risks, which are primarily resides in service providers, communicating channels or service receiver sides, can be well identified and managed using clinical risk management, M-Health and context aware systems. Thereby, these systems can offer qualified and precise services.
\end{abstract}

\section{Keywords}

Mobile Health (M-Health), Context Aware System, Clinical Risk Management

\section{Introduction}

Information Communication and Technology (ICT) today could have penetrated in many sciences, and have had 
good and positive effects on them. Medical science also could have received significant effects from ICT in the framework of its infrastructure. Today, issues such as Telehealth, Telemedicine, M-Health, etc., have a very fundamental role in delivering medical services to related stockholders. However, it seems that medical science cannot have used ICT capacities as it should be. One of the factors of this issue is sensitivity of this field that every decision and technology used in it has a direct relationship with people's health. So it is required that entrance of ICT and its durability in the field of medicine should be in such a way that does not cause increasing or happening of new problems in this field.

In this paper, we attempt to show the relationship among M-Health services, context aware systems, and clinical risk management process, by introducing $\mathrm{M}$-Health services, context aware systems and clinical risk management process and offering a general model of system and data flow model in it, in order to provide a solution for delivering appropriate services to patients and developing low cost solutions (in terms of financial aspects, etc.) for making a connection between patients and health centers and also for managing and detecting risks which can occur in these services or systems.

\section{Ease of Use}

\subsection{Mobile Health (M-Health)}

Patients sometimes are deprived of accessing various health services, because of problems such as patients' inability for going to long trips or financial problems and such problems. The key point is in decreasing costs of health care, continuing education for patients, medical management, and regular meetings with doctor. Obtaining confidence that patients receive adequate training for disease prevention appropriately, can prevent from many costs. The study conducted in Asheville area showed that chronic disease management and patients' training decrease health care costs $34 \%$ for each patient. Today, significant progress in the field of communication and mobile phone technology has opened new horizons to health systems managers. Today health managers have concluded that regarding spread of using intelligent mobile phones, they can use mobile phone systems for delivering appropriate services with least costs to various users. Some of these services are Medication Management Messaging and Appointment Reminder Messaging services [1]. As another example, patients can send their vital data including body temperature, blood pressure, and heartbeat, through mobile phones to their doctor, and ask the doctor's opinion about these data.

Therefore, M-Health as a subset of Telehealth, can be defined as using portable electronic devices having application for delivering health services and patient's information management [2]. M-Health can offer various services remotely in prevention, detection, control, and treatment of disease or in the conditions of disaster for a patient or an environment. Developments in the field of wireless networks and mobile phone equipment have resulted in emerging M-Health services (MHS). These services can be defined as various services of health care including health consultation, hospital discharge, and services based on place, which are deliverable through communication technologies and mobile phone network. For instance, when a patient is in a place that does not have possibility of accessing wireless network and is suffered a heart attack, he/she can ask MHS by pressing a specific key on his/her mobile phone, then medical center will receive his/her message and regarding his/her place, they will send help for him/her [3].

\subsection{Context Aware System}

In order to define a context aware system, and implement clinical risk management process on it, it is required to obtain more information about clinical risk management and context. To start, we should have information about context life cycle. Figure 1 shows this cycle.

At first, context aware systems discovered available context information providers (CIP). This discovery can be performed as pull or push [4]. For example, GPS receiver can also sent geographical information at each second to context aware systems (Pull) or context aware systems can ask GPS for geographical information at each second (Push). Then, context aware systems collect context information from discovered CIPs, and store them in context information repository for the next stage. Finally, reasoning mechanism enables programs to use advantages of available context information. Reasoning can be performed based on a piece of information or a set of information. For example, in a context aware system for electronic health, user's health status can be evaluated based on his/her heartbeat and blood pressure, which are sent through medical sensors [5]. 


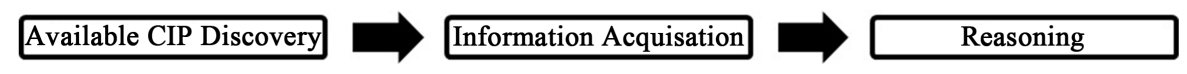

Figure 1. Context life cycle [4].

Information obtained from context, have some attributes. Generally, these attributes can be classified in three main categories of durability, origin, and quality. Durability specifies the relation between information and their variability over time. Clearly, static context information neither changes, nor changes very slowly. On the other side, dynamic information change with higher speed. Origin of context information can be external or internal. For example, in a program conducted in mobile phone, information results from mobile phone itself, are internal information, while information result from GMS operators are considered as external information. Distancing internal or external information in evaluation can be very important. The most important attribute of context information, is its quality. According to context information quality, obtaining information can repeat periodically. This repetition can be because of refreshing information or a specific event. Once classifying context information clearly, and collecting a list of appropriate attributes, the relationship between various kinds of context information should be identified in order that future reasoning about context becomes possible. Identifying the relationship between context information makes possible information gathering at the level of program [4].

Regarding the provided descriptions about context, one can consider a model for context. Preuveneers et al. provided a model for context as illustrated in Figure 2.

According to this model, context is composed of three components of user, environment, and execution environment. Environment includes temperature, light, sound, pressure, humidity, time, and place. User includes profile information, his/her emotional state, and execution environment includes software and hardware characteristics [6].

\subsection{Clinical Risk Management}

Once obtaining information about context, then we need to gather information about clinical risk management, too. Clinical risk management process can be defined as an approach for improving safety and quality of delivered health cares through paying special attention to identifying situations which expose patients to risk of injury, and acting or preventing or controlling these situations [7]. This process is a five-part process composed of monitoring, context development, risk identification, risk analysis, risk evaluation, and risk treatment [8].

At the stage of investigating and monitoring, in order to ensure that health risk management program is an appropriate program, continuous investigation and monitoring should be implemented on health risk management process. As effective factors on possibility of event and risk consequences can change continuously, continuous repetition of investigation and monitoring stage is necessary along risk management process [7] [9] [10].

At the stage of developing context, health service managers should identify action environment and strategic domain on health risk management process and understand it well. Although it is required that the environment in which the organization is acting, be defined well; therefore next steps toward health risk management process will have significant domain [7] [9] [10].

Comprehensive identification of risks which should be managed by using structured systematic process is a critical issue. During time, all health risks should be identified, evaluated, treated, and monitored. Although for beginning identification process and prorating health risks, which can result in threat, is a necessary issue. Various domains can be apt for occurring risk, that generally these domains can be divided into three categories of the service receiver environment, service provider environment, and communication channel (communication channel environment). There are various methods of identifying health risks that some of them are complaint data, work breakdown structure, operational modeling, flowchart drawing, investigating system plan, system analysis, system engineering techniques, including hazard and operability studies (HAZOP), survey or physical inspection, decision tree, surveys, questionnaire and Delphi technique, analyzing strengths, weaknesses, opportunities, and threats (SWOT) and brainstorm [7] [9] [10].

Analyzing risk includes considering available controls on health risk, degree of possibility of consequences which risk results in disaster, and degree of possibility of occurring these consequences. In order to be able to do a more suitable analysis of risk, one should consider their occurring possibility associated with their effect on project [7] [9] [10]. 


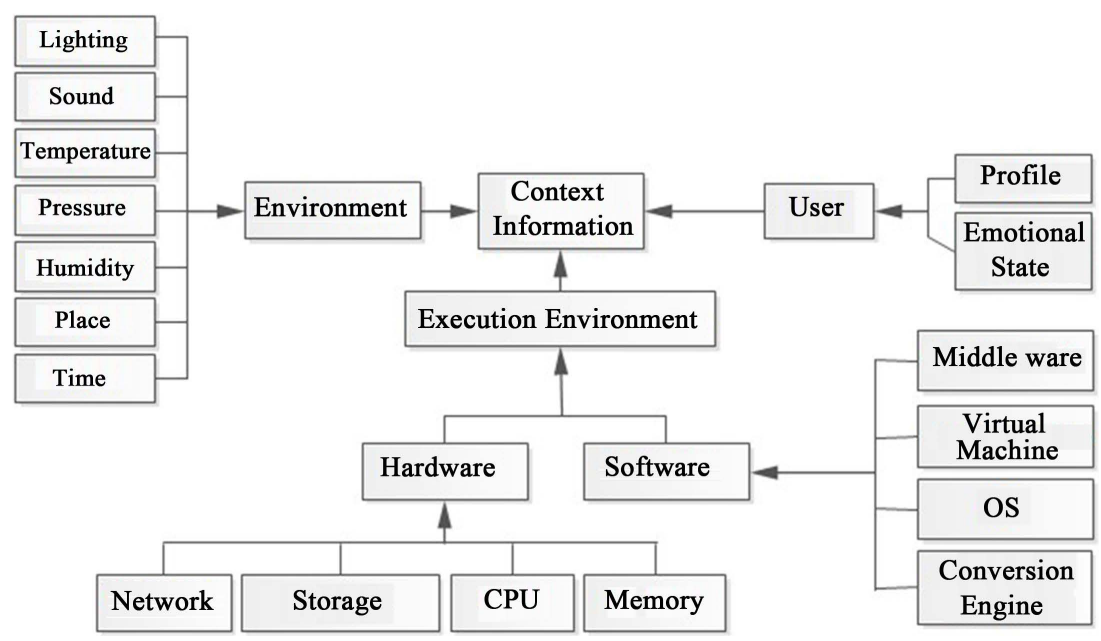

Figure 2. Context model provided by Preuveneers et al. [6].

Evaluating and prorating risk includes comparing risk level found along identifying process based on predetermined risk scale and developing priority list for future measures. Health services at first should investigate their identified health risks based on specified scales. If treatment risk be acceptable, this risk will not be treated at the stage of risk treatment. Although specifying risk as an acceptable risk does not consider it as a negligible health risk. Once evaluation scale is developed for health risk framework, health service should define acceptable health risk levels for organization in various internal and external environments. Risk scale is used for measuring and ranking risks and for specifying which risks are acceptable, and should managed at which organization level. While deciding about acceptability of health risk, importance of health risk and importance of policies, programs, processes, and activities should be under control. This risk evaluation should consider control level that organization has for each risk associated with potential financial effect and benefits and opportunities. Also, consequences and potential risks due to other stockholders should also be considered. If health risks be not acceptable for organization, then these risks should be treated. These health risks should then be prioritized for appropriate management under work plan and organization's strategic organization of health risk management [7] [9] [10].

At the stage of risk treatment, should also consider risk treatment including identifying domain of possible options for risk treatment, their evaluation, preparing risk treatment plans and their implementation. In treating health risk we are faced with alternatives for treating risks that were not acceptable at previous stages for the organization. Treatment alternatives available for health services include avoiding risk, decreasing risk level, transferring health risk, and maintaining health risk [7] [9] [10].

Regarding above provided explanations, the origin of available risks in delivering mobile health services by the help of context aware systems can be divided into three main categories. The first category relates to service provider environment. The second category relates to service receiver environment. The third category relates to communication channel between receiver and sender. It is obvious that in such a system, human force element and technology have a fundamental role, and need special consideration. In this respect, for better management of risks, we can refer to a framework which is composed of three different levels. At the first level, risks separate into human and technologic issues widely. This level is the basic level. The second level is specified to risks due to using mobile technology for supporting health cares. The third level is dedicated to risks due to technology failure or lack of reliable action of technology. Very damaging aspects of risk of defects in technology are communication networks, access equipment, monitoring equipment, and integration of information resources [11]. Some cases of various risks which can be existed in this category are [12]:

- Some risks in service provider part:

- Wrong regulation of context aware system parameters: applying inappropriate regulations for system parameters. For example, selecting inappropriate threshold amount for blood pressure.

- The plan of providing conflict services: it might be that a service providing plan declares a work for a specified time, and another service providing plan declares another work for the same time. For example, for 8 a.m. both doing patient's blood test and walking considered simultaneously. 
- Omission of service providing plan: may be providing service plan is omitted.

- Lack of information: because of reducing the relationship facing service receiver and service provider, may be service provider information be at the situation and condition of receiving low information.

- Some risks in service receiving part:

- Cheating or delivering wrong information in system: when there is a need of approval of service receiver, he/she delivers wrong information. For example, service receiver declares wrongly about drug consumption that consumption has been done.

- Some risks in service communicating channel part:

- Inappropriate treatment plan.

- Service failure: may be provided service by the third party of service providing, stop working or doesn't have correct function.

- Network hardware failure: may be because of any reason, network stop working in terms of its technics and hardware.

- Malicious actions by hackers: may be setting of parameters of context aware system or data of service receiver changed or stolen.

\section{Results}

In order to modeling M-Health services risk management associated with context aware systems, we should model three systems (Clinical risk management, M-Health, and context aware system), and extract flow of information in these three systems and also among these three systems. At the first stage, as we attempt to obtain a series of parameters from external environment and patients and processing these information in some specific units, we deliver decisions and required outputs to the final user, thus in summary we can state that we try to deliver an information system. For this aim, we can consider information process model in this system as the following:

As it is shown in Figure 3, in context aware system after identifying available CIPs are acted relative to obtaining context information through selected CIP, and finally reasoning about information is performed and based on this reasoning, services are provided to patients. In the meantime, clinical risk management system can influence all system from the results obtained from their internal process. As it is obvious in Figure 3, in addition to providing service based on the results obtained from context aware system reasoning, services in this system can be asked without considering context aware system; patients themselves can act toward asking service, and after investigating these requests, if service is confirmed, will be delivered to patients. Clinical risk management system is also effective at this stage.

We can investigate the desired system from a higher perspective, and then investigate relationship of this system with other systems.

As it is shown in Figure 4, the offered system is related with the following systems:

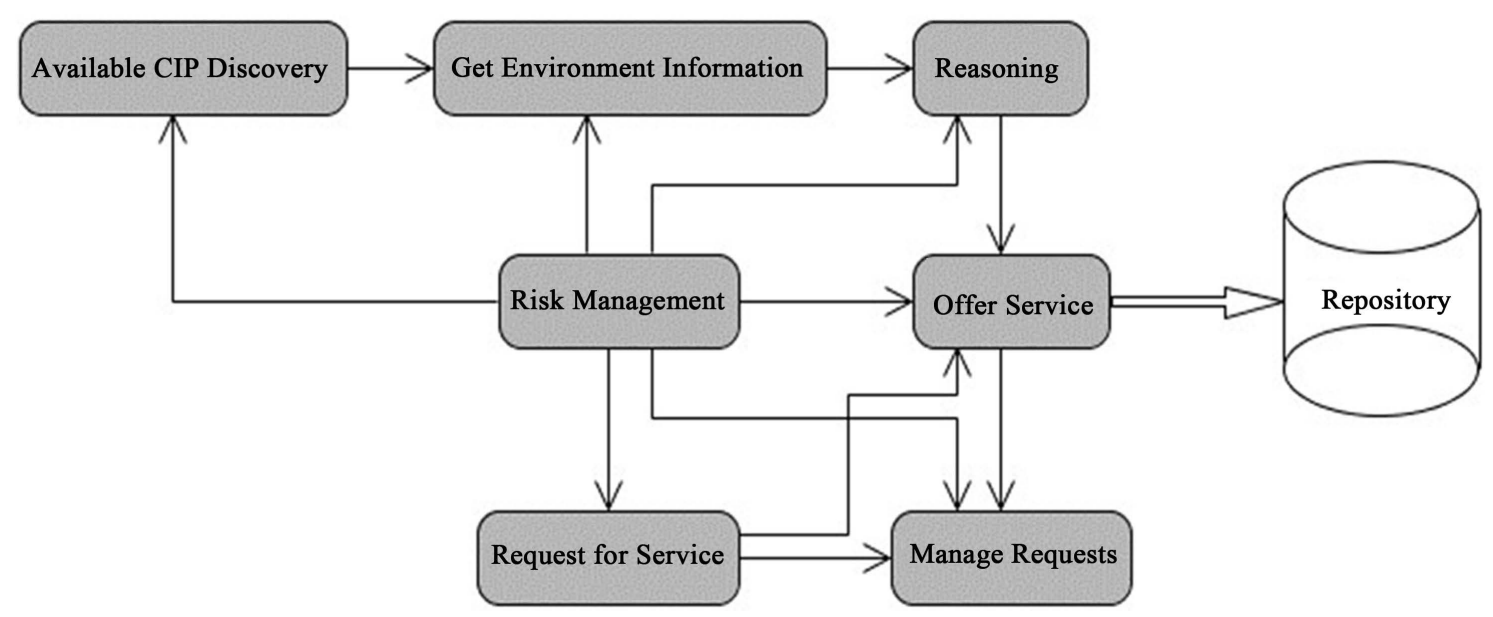

Figure 3. Information process model. 


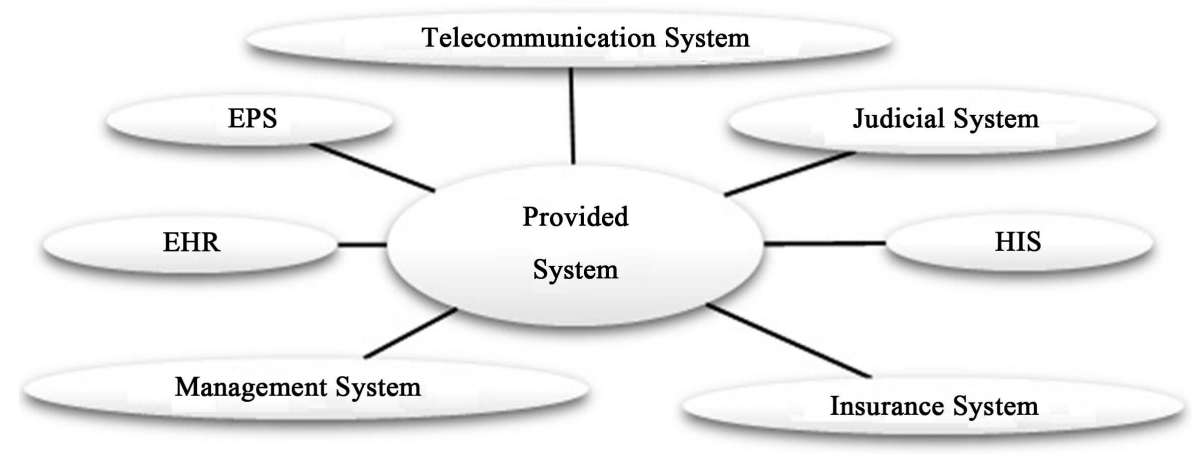

Figure 4. Context model.

Judicial Systems: These systems should be able to investigate provided services to patients in conditions of complaint occurrence. The relationship of these systems with the desired system is a one-way relation and these systems uses information of the desired system.

Hospital Information Systems (HIS): These systems should record services provided to patients in EPR, and works related to their financial affairs, etc. The relation of these systems with the desired system is a two-way relation. These systems also enter information in the desired system, and also uses of its information.

Insurance Systems: Insurance systems should be aware of services provided to patients in order to be able to act on its insurance obligations. The relation of these systems with the desired system is a one-way system, and these systems uses information of the desired system.

Management Systems: Management systems should be able to obtain management reports from provided services in order to be able to obtain appropriate management strategies. The relation of these systems with the desired system is a one-way relation and these systems uses information of the desired system.

Telecommunication Systems: Telecommunication systems should be able to provide services on time and with high quality. Sometimes it is required to use message sending and call systems, and sometimes it is also necessary that services transfer through other communicating channels including satellite and various antennas. The relation of these systems with the desired system is a one-way relation and the desired system uses information of this systems.

Electronic Prescription Systems (EPS): Any kind of service that is provided to patients, if it has prescriptive aspect, should also be recorded in this system that later have patient's health prescriptions and patient's treatment history. The relation of these systems with the desired system is a one-way relation and these systems uses information of the desired system.

Electronic Health Record Systems (EHR): These systems should be able to have the summary of provided services to patient to maintain patient's information integration. The relation of these systems with the desired system is a two-way relation. These systems also enter information into the desired system and also use them.

Now that we recognized system boundary, we can investigate data flow in this system. This flow can be portrayed in the form of Figure 5. It is obvious that the results obtained from clinical risk management process can influence the whole system. Also, delivering service in this system can be done in two ways:

1. Delivering service subject to doctor confirmation

2. Delivering service directly

Also, in this system we can consider three main repositories, which one is for electronic health record, and provided services to patient are sent to it for storage or management reports are extracted from it. The second repository relates to the history of requests in which all requests of patient (confirmed or rejected by doctor) are stored. The third repository relates to risk history in which risks are stored with the results obtained from their evaluation and treatment.

In this system, data flow is started either through context aware system (Character C), or through patient (Character $\mathrm{P}$ ). Data flow inside clinical risk management system is also shown by character $\mathrm{R}$. The numbers on each of the arrows show sequence of work. For example, at first the patient requests service (1.P), this request should be recorded in history repository of service request (2.P), then this request of service is sent to his/her doctor for investigation, and waits for investigating and confirming of doctor (3.P). If the doctor confirm delivered service 


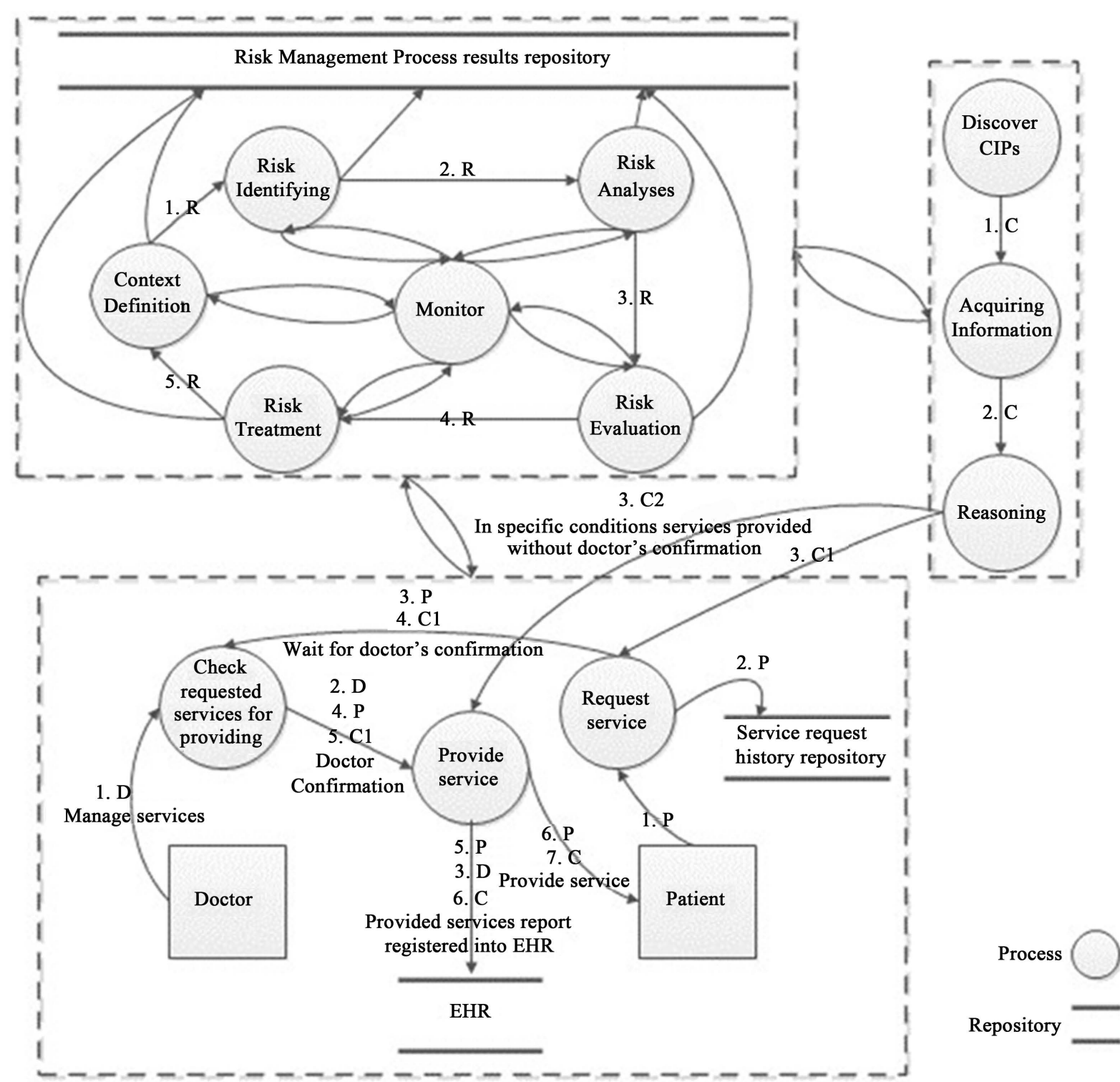

Figure 5. Data flow process.

(4.P), then service for patient is provided (5.P), and finally information of this service is recorded in electronic health record repository (6.P).

About system execution process, clinical risk management controls the whole system as an umbrella (service requester, service provider, and communication channel). Each stage of clinical risk management process relates to monitoring stage. Context aware system also by the help of various information received from environment, does its reasoning, and provides appropriate service. Generally, this system execution process can be shown in the form of Figure 6.

\section{Conclusion}

In this paper, after investigating M-Health, clinical risk management and context aware systems, information model and context model of the desired system were offered. In information model, it was shown that services in the desired system could be requested through context aware system and patients themselves. In context model, it was shown and explained that various systems could be related with the studied system. Finally, after describing these two models, data flow process and system execution process were offered. In data flow process, 


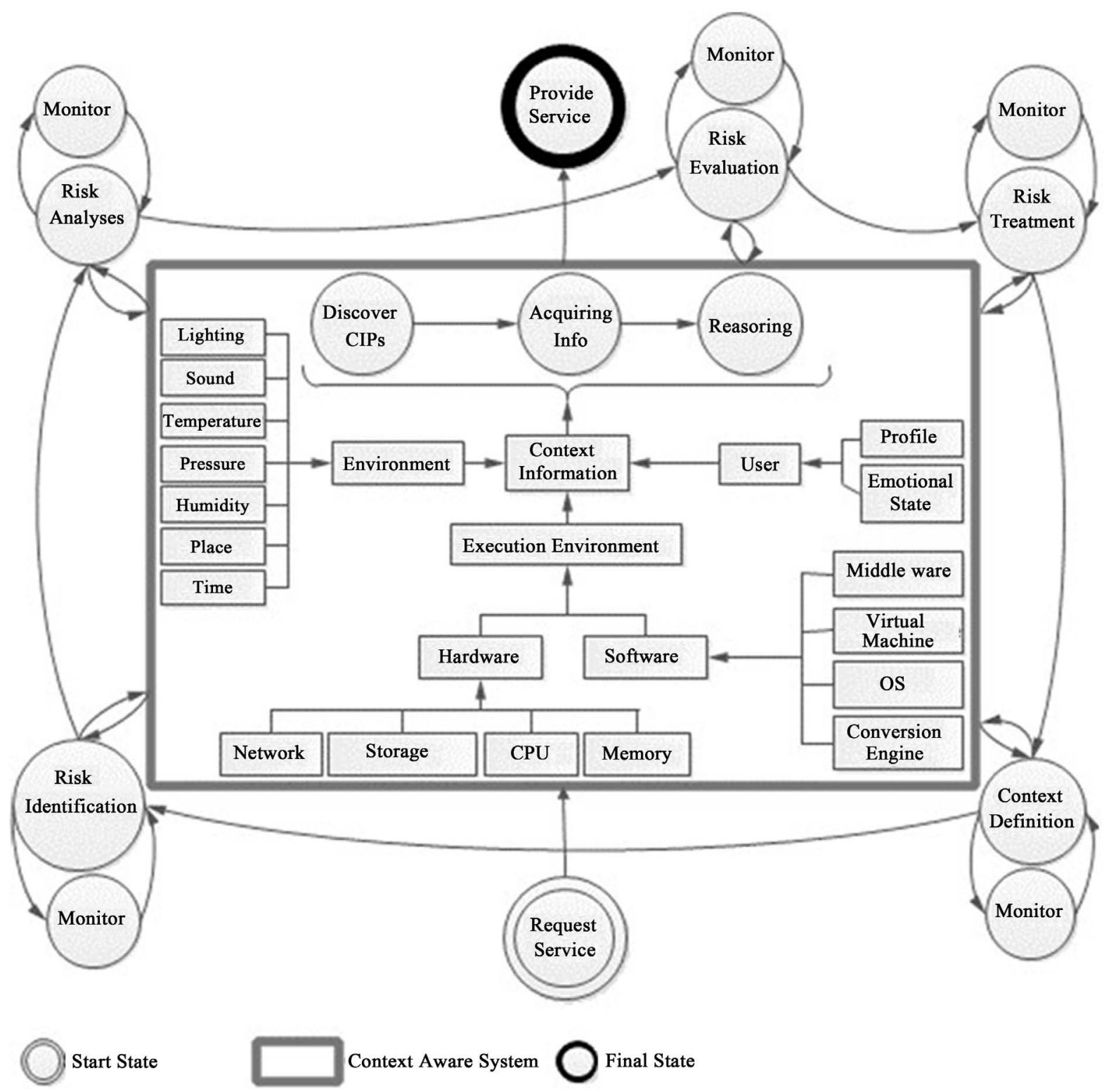

Figure 6. System execution process.

data movement and flow in system were shown. And finally in the offered executive process, attempts had been made to offer a model realistically, in order to be able to use this model for producing executive system. Also, in the offered executive process, relations among mobile health system, risk management and context aware system were portrayed.

\section{References}

[1] Trust, C. (2014) Mobile Healthcare Solution | Prevention and Intervention. http://www.celltrust.com/Solutions/HealthCare/CellTrust-Solutions-Healthcare-PI.html.

[2] Källander, K., Strachan, D.L. and Conteh, L. (2013) Mobile Health (mHealth) Approaches and Lessons for Increased Performance and Retention of Community Health Workers in Low- and Middle-Income Countries: A Review. Journal of Medical Internet Research. http://dx.doi.org/10.2196/jmir.2130

[3] Sun, Y., Wang, N., Guo, X. and Peng, Z. (2013) Understanding the Acceptance of Mobile Health Services: A Comparison and Integration of Alternative Models. Journal of Electronic Commerce Research, 14, 183-200. 
[4] Wrona, K. and Gomez, L. (2006) Context-Aware Security and Secure Context-Awareness in Ubiquitous Computing Environments. Annales UMCS, Informatica, Vol. 4. No. 1.

[5] Dey, A.K. and Abowd, G.D. (1999) Towards a Better Understanding of Context and Context-Awareness.

[6] Preuveneers, D., Bergh, J.V.D., Wagelaar, D., Georges, A., Rigole, P., Clerckx, T., et al. (1999) Towards an Extensible Context Ontology for Ambient Intelligence.

[7] Department of Health Government of Western Australia (2006) Clinical Risk Management Pocket Guide for the Western Australian Health System Information. Series No. 8.

[8] Asosheh, A. (2009) IT Project Management, Services-Based and Knowledge-Oriented.

[9] Care, C.H. (2004) Effective Health Care Risk Management Programs: Components for Success.

[10] Berg, H.-P. (2010) Risk Management: Procedures, Methods and Experiences. RT\&A, 2.

[11] Papadopoulos, H., Pappa, D. and Gortzis, L. (2007) A Framework for Dealing with Legal and Clinical Risks Arising from the Use of M-Health Systems. Journal on Information Technology in Healthcare, 5, 182-195.

[12] Eslami, M.Z., Sapkota, B., Zarghami, A., Vriezekolk, E., van Sinderen, M. and Wieringa, R. (2012) Risk Identification of Tailorable Context-Aware Systems: A Case Study and Lessons Learned. 